\title{
Test Results of LHC Interaction Regions Quadrupoles Produced by Fermilab
}

\author{
R. Bossert, J. Carson, D.R. Chichili, S. Feher, J. Kerby, M.J. Lamm, A. Nobrega, T. Nicol, \\ T. Ogitsu, D. Orris, T. Page, T. Peterson, R. Rabehl, W. Robotham, R. Scanlan, P. Schlabach, \\ C. Sylvester, J. Strait, M. Tartaglia, J.C. Tompkins, G. Velev, S. Yadav, A.V. Zlobin
}

\begin{abstract}
The US-LHC Accelerator Project is responsible for the production of the Q2 optical elements of the final focus triplets in the LHC interaction regions. As part of this program Fermilab is in the process of manufacturing and testing cryostat assemblies (LQXB) containing two identical quadrupoles (MQXB) with a dipole corrector between them. The $5.5 \mathrm{~m}$ long Fermilab designed MQXB have a $70 \mathrm{~mm}$ aperture and operate in superfluid helium at $1.9 \mathrm{~K}$ with a peak field gradient of 215 $\mathbf{T} / \mathrm{m}$. This paper summarizes the test results of several production MQXB quadrupoles with emphasis on quench performance and alignment studies. Quench localization studies using quench antenna signals are also presented.
\end{abstract}

Index Terms-Interaction Region, LHC, Low- $\beta$ Quadrupole, Superconducting Magnet.

\section{INTRODUCTION}

$\mathrm{H}$ IGH gradient final focus triplets quadrupoles are needed for the interaction regions at the Large Hadron Collider (LHC). Each of four detectors requires two final focusing triplets (Q1, Q2, Q3). The quadrupoles have a $70 \mathrm{~mm}$ bore and peak operating field gradient of $215 \mathrm{~T} / \mathrm{m}$. Nine triplets will be built, providing one spare.

Fermilab is building half of the interaction region inner triplet quadrupoles (Q2) and KEK the other half (Q1 and Q3). Final assembly and cryostating of all magnets is being done by Fermilab. Q1 and Q3 magnets each consist of $6.3 \mathrm{~m}$ long quadrupole plus correction elements. Q2 magnets consist of two $5.5 \mathrm{~m}$ long quadrupoles (MQXB) with a corrector element between them, all installed in a single cryostat (LQXB). Fermilab has built 15 production MQXB cold masses and 7 LQXB assemblies: LQXB01 - LQXB07. Six of these assemblies have been tested at the Magnet Test Facility in Fermilab. The seventh assembly is ready to be tested.

This paper summarizes the test results of LQXB01-6 focusing on magnet quench performance and alignment issues. Magnetic measurements and details of the cold mass

Manuscript received October 20, 2003.

This work was supported in part by the U.S. Department of Energy.

T. Ogitsu is with KEK High Energy Accelerator Research Organization, Tsukuba, Ibaraki, 305-0801, Japan,. R. Scanlan is with LBNL Lawrence Berkeley National Laboratory, 1 Cyclotron Road, Berkeley, California 9472, rest of the authors are with FNAL Fermi National Accelerator Laboratory, P.O. Box 500, Batavia, Illinois 60510. and cryostat design can be found elsewhere [1] - [4].

\section{LQXB03-7 PRODUCTION HighLIGHTS}

Each LQXB consists of two cold masses, labeled Q2a and Q2b. All the cold masses in the production series were built in a nearly identical manner [5]. Coils were uniform in size and modulus of elasticity. All production quadrupoles were within the prescribed mechanical and electrical specifications.

There were no significant problems or design modifications during the fabrication of MQXB06-15. Early in the production run (during the construction of MQXB01-5), there were some problems during assembly with ground shorts, resolved beginning with MQXB05 [6].

Although there were no significant design changes during the production of the MQXB assemblies, there were some minor variations. Beginning with MQXB04, inner coils were shimmed by $38 \mu \mathrm{m}$ toward the midplane to adjust $\mathrm{b}_{6}$ harmonics. Also, coils were wound with cable containing strand produced by several different manufacturers, as shown in Table I.

TABLE I

COLD MASS STRAND MANUFACTURERS

\begin{tabular}{ccccc}
\hline \hline \multirow{2}{*}{ Q2 No. } & & Cold Mass No. & Inner strand & Outer strand \\
\hline \multirow{2}{*}{ LQXB01 } & Q2a & MQXB02 & Alstom & Alstom \\
& Q2b & MQXB01 & Alstom & Alstom \\
LQXB02 & Q2a & MQXB03 & Alstom & Alstom \\
& Q2b & MQXB04 & Alstom & Alstom \\
LQXB03 & Q2a & MQXB06 & Alstom & Alstom \\
& Q2b & MQXB05 & Alstom & Alstom \\
LQXB04 & Q2a & MQXB10 & Alstom & Alstom \\
& Q2b & MQXB12 & Oxford & Alstom \\
LQXB05 & Q2a & MQXB11 & Alstom & Alstom \\
& Q2b & MQXB08 & Alstom & Alstom \\
LQXB06 & Q2a & MQXB07 & Alstom & Alstom \\
& Q2b & MQXB09 & Alstom & Alstom \\
LQXB07 & Q2a & MQXB14 & Oxford & Alstom \\
& Q2b & MQXB15 & IGC & Alstom \\
\hline \hline
\end{tabular}

\section{TEST DETAILS}

\section{A. Quench Antenna}

The test stand is instrumented with a series of quench antenna panels on the inner surface of the vacuum space of the warm 
finger installed in the beam tube of the magnet during testing. These panels have precision traces etched on a Kapton substrate that when wrapped on the inner tube of the warm finger form a multipole winding sensitive to particular harmonics of the magnetic field. Details of the quench antenna are described elsewhere [6], [7].

\section{B. Alignment Measurements}

The Single Stretched Wire (SSW) system developed at Fermilab [8], [9] is used for all alignment measurements of the LQX magnets. A fine $(100 \mu \mathrm{m}) \mathrm{CuBe}$ wire is stretched through the entire length of the magnet, connected to precision $(1 \mu \mathrm{m}$ accuracy) motion stages at both ends, with the return wire of the flux loop lying fixed on the bottom of the beam pipe. Using co- and counter- directional stage motions (i.e. stages moving in the same or opposite directions), the $x, y, z$ and roll, pitch, yaw axes of each of the MQXB cold masses in the LQXB can be determined. Removal of sag effects from the $18 \mathrm{~m}$ wire used for measurements are achieved at the $1 \%$ level by measuring the vibration frequency at various tensions and extrapolating to infinite frequency (infinite tension). Powering is done AC for the warm measurements, and, except for the first magnet, also for alignment tests cold (in order to minimize the time needed for changeover of the high-current power leads). Roll measurements performed cold are done with DC current and Q2a and Q2b powered in series. A laser tracker is used to transfer fiducial positions of the stage to the external fiducials of the magnet. Precise calibration of the distances from the stage fiducials to the actual support point of the wire are obtained using an optical gauging microscope.

\section{QUENCH PERFORMANCE}

Up to date six LQXB assemblies went through a test cycle. All of the cold masses were tested separately. Three of them

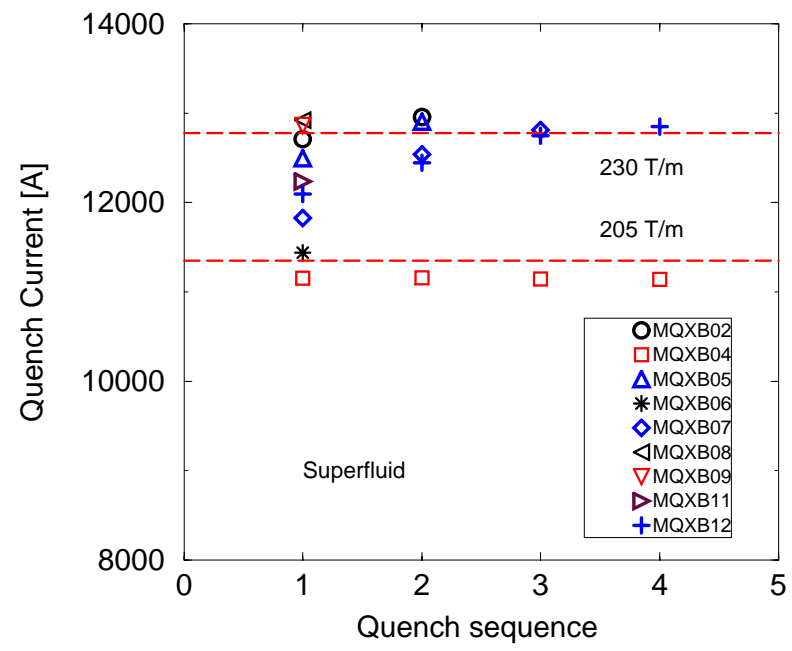

Fig. 1. Quench training plot for nine production magnets. $20 \mathrm{~A} / \mathrm{s}$ nominal ramp rate was applied. All of the production magnets exhibited very little training to achieve the planned quench current value which corresponds to $230 \mathrm{~T} / \mathrm{m}$.

(MQXB01, MQXB03, MQXB10) reached $13 \mathrm{kA}$ without quenching (20A/s nominal ramp rate was applied). The other magnets but MQXB04 exhibited very little training to reach the desired $230 \mathrm{~T} / \mathrm{m}$ field gradient value (see Fig. 1). MQXB04 however, has quenched below the required $205 \mathrm{~T} / \mathrm{m}$ operating field gradient value and showed no sign of any training. Using quench antenna signals the quench results can be explained by localized conductor damage [6]. In order to find the cause of the quench performance limitation, MQXB04 has been disassembled and the coils were examined carefully. The local conductor damage was not verified either by visual inspection or by testing the suspected cable segments separately.

Quadrant coil voltage taps and quench antenna signals were used to localize quenches. Table II. summarizes the quench locations. Some of the quench files were not saved consequently we were not able to localize quenches for those events. Quench antenna signals were only useful for

TABLE II QUENCH LOCATIONS

\begin{tabular}{|c|c|c|c|c|c|}
\hline Q2 & Cold mass & $\begin{array}{c}\text { Quench } \\
\text { No. }\end{array}$ & $\begin{array}{l}\text { Quench } \\
\text { Current }\end{array}$ & $\begin{array}{c}\text { Qudrant } \\
\text { No. }\end{array}$ & Location \\
\hline LQXB0 & MQXB04 & 1 & 11152A & Q2 & \\
\hline \multirow[t]{3}{*}{2} & & 2 & 11158A & Q2 & \\
\hline & & 3 & $11146 \mathrm{~A}$ & Q2 & LE1 \\
\hline & & 4 & 11142A & Q2 & LE1 \\
\hline LQXB0 & MQXB05 & 1 & $12495 \mathrm{~A}$ & Q2 & LE2 \\
\hline 3 & & 2 & $12905 \mathrm{~A}$ & Q2 & LE2 \\
\hline LQXB0 & MQXB12 & 1 & 12097A & Q2 & LE1 \\
\hline \multirow[t]{3}{*}{4} & & 2 & $12445 \mathrm{~A}$ & Q1 & LE2 \\
\hline & & 3 & $12749 \mathrm{~A}$ & Q3 & LE2 \\
\hline & & 4 & $12850 \mathrm{~A}$ & Q2 & BO2-BO1 \\
\hline LQXB0 & MQXB11 & 1 & $12236 \mathrm{~A}$ & Q3 & \\
\hline \multirow[t]{2}{*}{5} & & 2 & $12974 \mathrm{~A}$ & Q4 & BO1-LE3 \\
\hline & MQXB08 & 1 & $12922 \mathrm{~A}$ & Q3 & LE2 \\
\hline LQXB0 & MQXB07 & 1 & 11827A & & \\
\hline \multirow[t]{3}{*}{6} & & 2 & $12538 \mathrm{~A}$ & Q3 & LE2 \\
\hline & & 3 & 12812A & Q3 & LE2 \\
\hline & MQXB09 & 1 & $12864 \mathrm{~A}$ & Q1 & BO3 \\
\hline
\end{tabular}

LE1 => Lead End panel 1; BO1 Body Panel 1 (for details of the quench antenna description see [6])

localization when the quenches were within the sensitive area of the quench antenna windings. Most of the quenches appeared close to the lead end of the magnet but there were no indication that any particular location was favored (except for MQXB04) by the quenches.

\section{Alignment}

Alignment measurements are critical for installation of the LQXB assemblies in the LHC beam lines. Specified tolerances must be met for the relative positioning of the cold masses within the cryostat, and the actual cold mass positions must be measured and transferred to the external fiducials which are used during magnet installation in the tunnel. Furthermore, alignment stability is needed over thermal cycles and transport.

A summary of cold Q2a/Q2b alignment data is given in Table III. For each of these measurements, the wire end points have been placed on the average axis of the Q2a and Q2b elements marking the location where the beam would pass through a net zero field (i.e. the net transverse center offsets from this line are zero). These are the positions transferred to 
the external fiducials which are to be used for magnet placement in the tunnel. The average misalignment of the individual magnet ends from the Q2aQ2b combined 'zeroaxis' is $0.15 \mathrm{mrad}( \pm 0.4 \mathrm{~mm})$ with standard deviation $0.11 \mathrm{mrad}$ $( \pm 0.3 \mathrm{~mm})$. These are within the required tolerances for LHC operation [10].

TABLE III

Q2A/Q2B ANGLES ON AVERAge AXIS

\begin{tabular}{ccccc} 
& Q2a Yaw & Q2a Pitch & Q2b Yaw & Q2b Pitch \\
\hline LQXB01 & 0.03 & -0.04 & 0.20 & -0.27 \\
LQXB02 & 0.21 & 0.01 & 0.13 & 0.19 \\
LQXB03 & -0.52 & 0.23 & 0.05 & 0.20 \\
LQXB04 & -0.07 & -0.09 & -0.04 & -0.10 \\
LQXB05 & -0.15 & -0.04 & -0.08 & -0.05 \\
LQXB06 & -0.12 & -0.23 & 0.21 & -0.26 \\
\hline
\end{tabular}

Measured yaw and pitch of the Q2a and Q2b elements when on axis passing through their centers (units are mrad). Note that $0.1 \mathrm{mrad}$ yaw/pitch corresponds to $\pm 0.275 \mathrm{~mm}$ at each end of the $5.5 \mathrm{~m}$ magnet length.

TABLE IV

Q2A/Q2B ANGLE CHANGES COLD-WARM

\begin{tabular}{lrrrrr} 
& Q2a Yaw & Q2a Pitch & Q2b Yaw & Q2b Pitch & Ave. Roll \\
\hline LQXB01 & 0.10 & -0.14 & 0.06 & 0.06 & 0.06 \\
LQXB02 & 0.30 & 0.01 & 0.33 & 0.47 & -0.13 \\
LQXB03 & -0.14 & 0.10 & -0.03 & 0.14 & -0.09 \\
LQXB04 & 0.03 & -0.08 & -0.06 & 0.05 & 0.03 \\
LQXB05 & -0.03 & -0.16 & -0.17 & 0.01 & -0.30 \\
LQXB06 & 0.08 & -0.17 & 0.08 & 0.07 & -0.15 \\
\hline
\end{tabular}

Measured change in yaw and pitch angles of Q2a and Q2b during thermal cycle from room to cryogenic temperature (units are mrad). Note that $0.1 \mathrm{mrad}$ yaw/pitch corresponds to $\pm 0.275 \mathrm{~mm}$ at each end of the $5.5 \mathrm{~m}$ magnet length.

Table IV gives the absolute warm/cold change in angles observed from the fixed reference frame of the fiducials. The changes reported are from the last cold measurement to the subsequent warm measurement. The warm/cold changes in the average centers are shown in Table $\mathrm{V}$ and are also plotted in Fig. 2. Warm/cold change in the average centers is generally less than $0.3 \mathrm{~mm}$.

TABLE V

\begin{tabular}{crrrr}
\multicolumn{5}{c}{ Q2A/Q2B XY-CENTER CHANGES COLD-WARM } \\
& Q2a X & Q2a Y & \multicolumn{1}{c}{ Q2b X } & Q2b Y \\
\hline LQXB01 & 0.01 & -0.26 & -0.16 & -0.15 \\
LQXB02 & -0.81 & -1.28 & -0.32 & -0.05 \\
LQXB03 & -0.15 & 0.07 & 0.15 & -0.14 \\
LQXB04 & 0.00 & 0.19 & -0.02 & -0.06 \\
LQXB05 & 0.23 & 0.31 & -0.10 & -0.10 \\
LQXB06 & -0.15 & 0.26 & -0.11 & -0.13 \\
\hline
\end{tabular}

Measured change in average centers for the individual Q2a and Q2b elements during thermal cycle from room to cryogenic temperature (units are mm).

It is interesting to note that the Q2a and Q2b elements seem to show paired motion which is either vertical, or having slope which is roughly consistent with \pm 45 degrees - the angle at which the cold mass support brackets are attached to the cryostat.

\section{LQXB Cold-Warm Average Center Change}

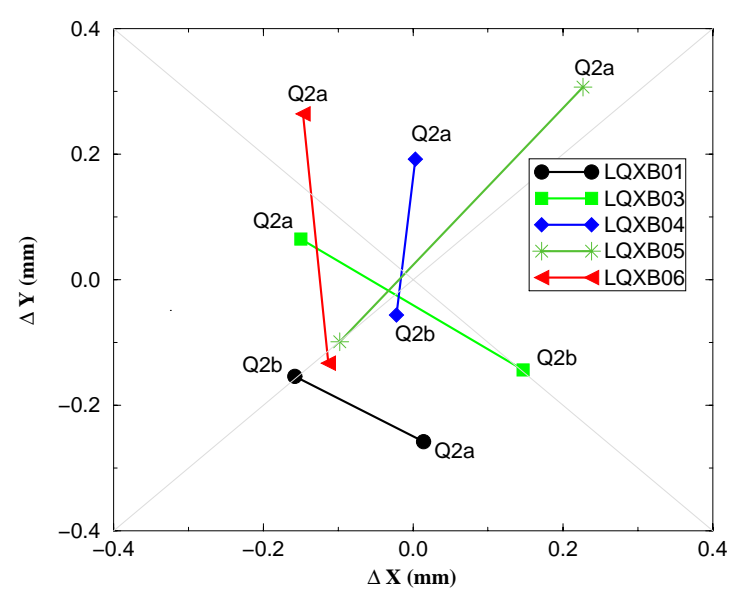

Fig. 2. Motion of average centers for the individual cold masses during cool-down from room to cryogenic temperatures. Note that data from LQXB02 have been suppressed for readability.

Warm/cold changes are not problematic if they are reproducible across multiple thermal cycles. It was observed, however, that the cold mass positions on the first cool-down of each magnet do not reverse upon warm-up. Fig. 3. relates the change in alignment parameters from warm before initial cool-down to warm after the thermal cycle (averages are shown with open circles and error bars). Some parameters such as Y-offset in both Q2a/Q2b and to a lesser extent, all the Q2a parameters, show average systematic changes across the first thermal cycle. Also shown in the figure are the second thermal cycle of LQXB01, and the second and third thermal cycles of LQXB03. The changes for both 'TC2' measurements are better, but still suggest that changes are not quite stable at the level of $0.25 \mathrm{~mm}$. The TC3 data for LQXB03 indicate stability has been reached.

\section{Warm-Warm Change Across Thermal Cycles}

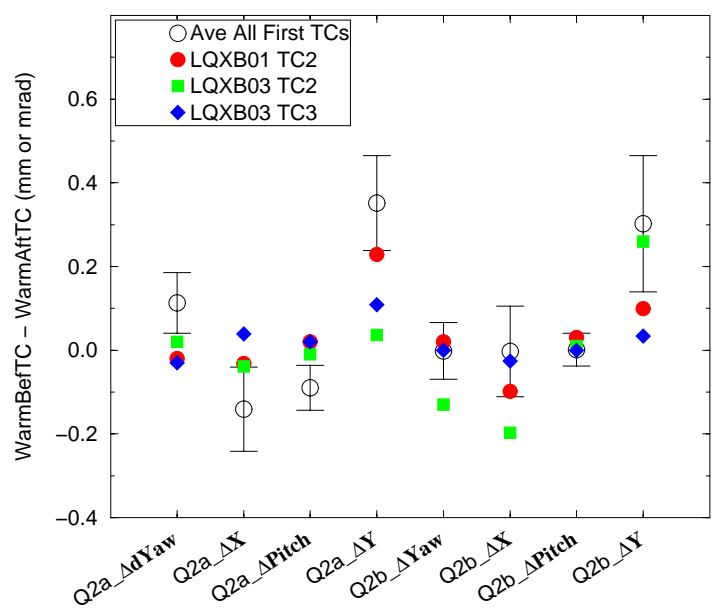

Fig. 3. The average change in alignment parameters over all magnets from warm before first thermal cycle (TC) to warm after first TC are shown with open circles. Subsequent TCs are shown for those magnets in which they are available. Changes are given in mm or mrad as appropriate. 
Adjustment lugs for the Q2a and Q2b cold masses are accessible through vacuum ports on the cryostat. These are adjusted to achieve as best alignment as possible before the initial cool down. An example of this is shown in Fig. 4 for LQXB04.

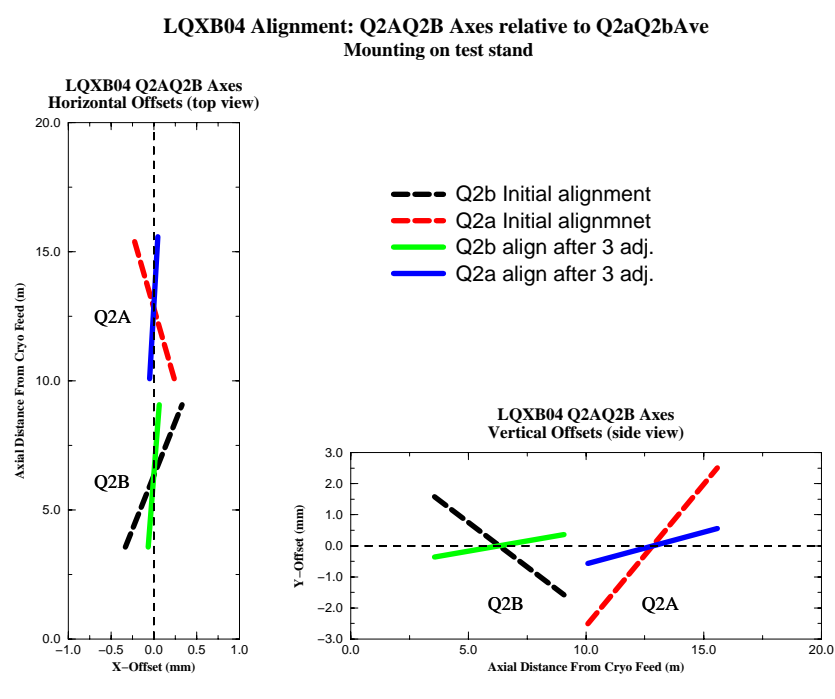

Fig. 4. Cold mass re-positioning in cryostat using adjustment lugs before initial cool-down.

Based on the warm/cold change that occurs during testing, and the resulting cold position, the alignment can be further tuned via lug adjustment after cold tests. Fig. 5 shows the effects of making changes to the alignment lugs in LQXB01 after its initial cool-down. The change in axes positions is measured warm. The magnet is then cooled-down and

LQXB01 Mechanical Change Correlation

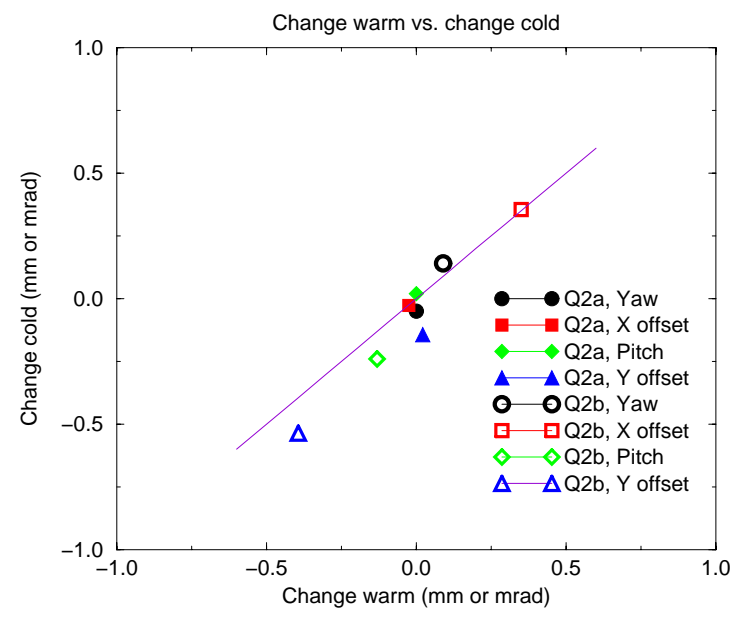

Fig. 5. Correlation between warm measurements of alignment changes after lug adjustment and the change observed during cold measurements. The line represents perfect correlation.

measurements of change in axes positions are repeated cold. The change observed cold is quite close to the change expected from the warm data, lending confidence that tuning of final cold-mass positions performed warm leads to predictable changes in the cold positions.

\section{CONCLUSIONS}

Quench performance for most of the cold masses was excellent. Three cold masses reached $230 \mathrm{~T} / \mathrm{m}$ without any quench and all the others have been trained within five quenches. Quench locations tend to occur close to lead end of the magnet but no indication for favored spots.

Alignment of the LQXB magnets has been measured, and relative alignment of the Q2a and Q2b elements are within tolerances required. Warm-cold changes are observed, especially during the initial cool-down; the magnitude of these is generally less than $0.3 \mathrm{~mm}$.

\section{ACKNOWLEDGMENT}

The authors thank the staff of Fermilab's Technical Division who have worked so hard on this effort over the course of the past year.

\section{REFERENCES}

[1] R. Bossert et al., "Development of a high gradient quadrupole for the LHC interaction regions", IEEE Trans. on Applied Superconductivity, Vol. 7, No 2, June 1997, p. 751.

[2] R. Bossert et al., "Fabrication of the first short model of high gradient quadrupole for the LHC interaction regions", MT-15 Proceedings, Beijing, October 1997, p. 191.

[3] N. Andreev et al.,"Quench performance of Fermilab high gradient quadrupole short models for the LHC interaction regions”, PAC'99, New York, April 1999, p. 3197.

[4] G.V. Velev, "Field Quality Measurements of the LQXB Inner Triplet Quadrupoles for LHC”, submitted to this conference.

[5] R. Bossert et al., "Costruction Experience With MQXB Quadrupole Magnets Built at Fermilab for the LHC Interaction Region” , IEEE Trans. Applied Superconductivity,vol. 13, No.2, June 2003, pp.12971300

[6] R. Bossert et al., "Test Results from the LQXB Quadrupole Production Program at Fermilab for the LHC Interaction Regions “,MT-18 Proceedings, October 2003,p.

[7] T. Ogitsu, et al., "Quench Antenna for Superconducting Particle Accelerator Magnets” IEEE Trans. Magnetics, vol 30, 1964, pp. 22732276.

[8] J. DiMarco et al., "Field Alignment of Quadrupole Magnets for the LHC Interaction Regions", IEEE Transactions on Applied Superconductivity, Vol. 10, No. 1, March, 2000.

[9] J. DiMarco et al., "Alignment of Production Quadrupole Magnets for the LHC Interaction Regions”, ASC2002 Proceedings, 2002.

[10] T. Sen, private communication. See also "Alignment Tolerances of the IR Quadrupoles in the LHC”, FERMILAB-CONF-99/304, Nov., 1999. 